\title{
Self-Reconfigurable Micro-Implants for Cross-Tissue Wireless and Batteryless Connectivity
}

\author{
Mohamed R. Abdelhamid, Ruicong Chen, Joonhyuk Cho, \\ Anantha P. Chandrakasan, Fadel Adib \\ Massachusetts Institute of Technology \\ $\{$ mrhamid,raychen,joonhyuk, anantha,fadel $\} @$ mit.edu
}

\begin{abstract}
We present the design, implementation, and evaluation of $\mu$ medIC, a fully-integrated wireless and batteryless micro-implanted sensor. The sensor powers up by harvesting energy from RF signals and communicates at near-zero power via backscatter. In contrast to prior designs which cannot operate across various in-body environments, our sensor can self-reconfigure to adapt to different tissues and channel conditions. This adaptation is made possible by two key innovations: a reprogrammable antenna that can tune its energy harvesting resonance to surrounding tissues, and a backscatter rate adaptation protocol that closes the feedback loop by tracking circuitlevel sensor hints.

We built our design on millimeter-sized integrated chips and flexible antenna substrates, and tested it in environments that span both in-vitro (fluids) and ex-vivo (tissues) conditions. Our evaluation demonstrates $\mu$ medIC's ability to tune its energy harvesting resonance by more than $200 \mathrm{MHz}$ (i.e., adapt to different tissues) and to scale its bitrate by an order of magnitude up to $6 \mathrm{Mbps}$, allowing it to support higher data rate applications (such as streaming low-res images) without sacrificing availability. This rate adaptation also allows $\mu$ medIC to scale its energy consumption by an order of magnitude down to 350 nanoWatts. These capabilities pave way for a new generation of networked micro-implants that can adapt to complex and time-varying in-body environments.
\end{abstract}

\section{CCS CONCEPTS}

- Hardware $\rightarrow$ Full-custom circuits; • Computer systems organization $\rightarrow$ Sensor networks; • Applied computing $\rightarrow$ Life and medical sciences;

\section{KEYWORDS}

In-body IoT, Backscatter Communication, Wireless, Energy Harvesting, Batteryless, Reprogrammable

\section{ACM Reference Format:}

Mohamed R. Abdelhamid, Ruicong Chen, Joonhyuk Cho, Anantha P. Chandrakasan, Fadel Adib . 2020. Self-Reconfigurable Micro-Implants for CrossTissue Wireless and Batteryless Connectivity. In The 26th Annual International Conference on Mobile Computing and Networking (MobiCom '20),

\section{(c) (i)}

This work is licensed under a Creative Commons Attribution International 4.0 License. MobiCom '20, September 21-25, 2020, London, United Kingdom

(C) 2020 Copyright held by the owner/author(s).

ACM ISBN 978-1-4503-7085-1/20/09.

https://doi.org/10.1145/3372224.3419216

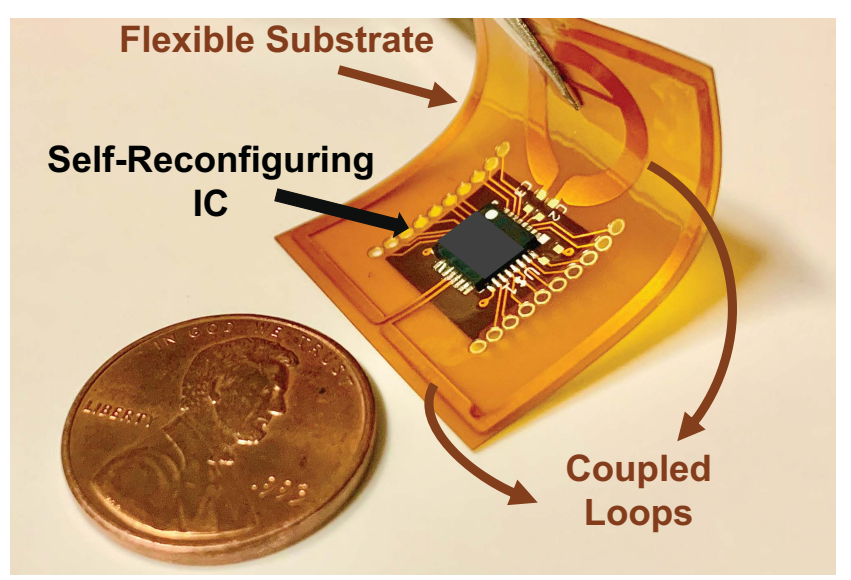

Figure 1- $\mu$ medIC. The self-reconfiguring hardware is implemented on an IC that controls the bi-resonance design on a flexible substrate. The penny is shown next to the micro-implant to demonstrate the form factor. $\mu$ medIC's flexible, thin design allows folding into an ingestible capsule or laminating it on an organ.

September 21-25, 2020, London, United Kingdom. ACM, New York, NY, USA, 14 pages. https://doi.org/10.1145/3372224.3419216

\section{INTRODUCTION}

The mobile networking community has recently witnessed mounting interest in wireless and batteryless sensors that can operate inside the human body $[22,33,54,63]$. These sensors can power up by harvesting energy from RF (Radio Frequency) signals transmitted from outside the body, and they communicate at near-zero power via backscatter - i.e., by reflecting existing signals rather than transmitting their own carrier. The combination of energy harvesting and backscatter communication allows these sensors to be batteryless. Independence of batteries eliminates the need for surgical replacement, allows ultra-long term operation, and enables miniature, fully-integrated form factors [35]. As a result, such sensors could be used for continuous monitoring of biomarkers and tumors, ultra-long lasting drug delivery systems (e.g., for patients with Alzheimer's or Osteoporosis), and closed-loop control systems with real-time feedback (e.g., artificial pancreas for Diabetes' patients).

A key challenge that faces existing solutions for wireless and batteryless micro-implants lies in their rigid designs which cannot adapt to different tissues or to time-varying in-body conditions. This is particularly problematic for mobile sensors like ingestible capsules, which experience a variety of in-body environments as they travel through the digestive tract to deliver drugs or sense biomarkers. The ability to adapt to different in-body environments is also key to enabling these sensors to operate across different humans, whose bodies have different tissue compositions (fat, muscles, etc.). The 
majority of existing solutions side-step this challenge by limiting themselves to shallow depths (i.e., on the body or right under the skin) $[22,53,63]$, where the ability to harvest energy is less impacted by the surrounding environment. Recent proposals (like IVN and ReMix) have tried to operate at larger depths at the expense of isolating the sensor (e.g., placing it in a test tube surrounded by air before implanting it inside tissues) [33] or by giving up energy harvesting altogether [54].

We present $\mu$ medIC, a fully-integrated wireless and batteryless sensor that can adapt to varying in-body conditions and can be directly integrated with tissues. Similar to past proposals, $\mu$ medIC harvests energy from RF signals to power up and adopts backscatter to enable energy-efficient communication. In contrast to past proposals, $\mu$ medIC introduces multiple innovations that allow it to adapt its energy harvesting to surrounding tissues and its communication throughput to in-body conditions. Moreover, $\mu$ medIC's design is implemented on an IC and flexible antenna substrate; this design allows rolling it into the form of an ingestible capsule or laminating it on tissues (e.g., on the stomach wall), enabling intimate integration with the human body.

Before we describe how $\mu$ medIC operates, let us understand why it is difficult for batteryless in-body sensors to operate across different in-body environments. Consider a sensor that needs to power up and communicate in the $900 \mathrm{MHz}$ ISM band, which is known to be optimal for energy-harvesting micro-implants [40]. ${ }^{1}$ In order to optimize energy harvesting, micro-implants are typically designed to resonate around the desired frequency of operation. The resonance frequency is determined by the shape of the antenna as well as the surrounding tissues (specifically, the dielectric of tissues in its immediate vicinity) [37]. Unfortunately, due to dependence on surrounding tissues, if a micro-implant is designed to resonate at $900 \mathrm{MHz}$ in a certain tissue (e.g., muscle), its resonance shifts to a different frequency (e.g., 1.1 GHz) when placed in another tissue (e.g., fat). Fig. 2 shows this problem by plotting the harvested voltage as a function of frequency for fat (red plot) and muscle (blue plot), which exhibit different resonance frequencies (peaks). This makes it infeasible to design batteryless micro-implants that can harvest energy across different tissues or maintain reliable backscatter communication if they need to travel through the human body. Furthermore, transmitting at frequencies outside the ISM band (e.g., around $1.1 \mathrm{GHz}$ ) to power up the sensor would make the system incompliant with FCC regulations for consumer electronics.

At the heart of $\mu$ medIC's approach is a programmable "coupled" antenna design. Antenna coupling is a well-known phenomenon and refers to the interaction between two antennas when they are close to each other. Because coupling alters the antenna resonance, it is generally considered harmful [57], and communication engineers typically try to separate antennas from each other to minimize coupling. In contrast, $\mu$ medIC employs coupling in order to control the resonance and adapt it to surrounding tissues. The design consists of two antenna loops (as shown in Fig. 1): an inner circular loop and outer rectangular loop. Because the two loops are in close proximity, they "couple" with each other, resulting in a resonance frequency

\footnotetext{
${ }^{1}$ In contrast, the MICS band around $400 \mathrm{MHz}$ is used for larger battery-powered implants such as cardiac pacemakers.
}

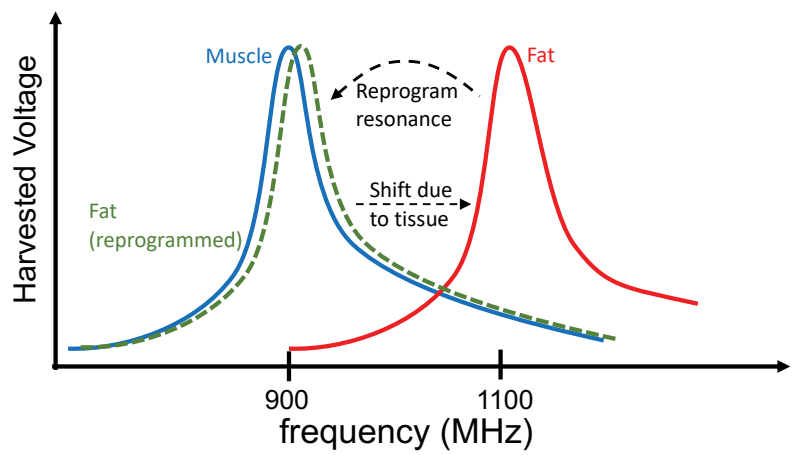

Figure 2-Reprogramming Resonance. The figure plots the harvested voltage versus frequency for a micro-implant placed in fat (red) and muscle (blue), demonstrating a shift in the resonance frequency. By reprogramming its resonance, $\mu$ medIC can move the resonance frequency back to $900 \mathrm{MHz}$ allowing efficient harvesting and communication.

that depends on both. $\mu$ medIC can reprogram this resonance by tuning a capacitive load on both the outer loop and the inner loop. For example, it can leverage this property to reprogram the resonance in muscle back to the $900 \mathrm{MHz}$ band as depicted by the green plot in Fig. 2. In $\S 4$, we describe this approach in detail, the rationale for antenna design, as well as how $\mu$ medIC's bi-loop design allows it to reprogram both the antenna and the matching hardware.

So far, we have assumed that $\mu$ medIC knows its surrounding environment (i.e., tissue composition and channel conditions) and can choose the best configuration to match that environment. However, in practice, such information is not available and difficult to predict. To deal with this uncertainty, $\mu$ medIC exploits circuit-level hints to perform rate and resonance adaptation. At a high level, it senses the harvested energy (voltage) and uses it for rate adaptation. $\mu$ medIC conservatively starts with a high-efficiency configuration and gradually increases its throughput. In $\S 5$, we describe this protocol in detail and show how $\mu$ medIC leverages low-level sensor hints to close the loop on rate adaptation.

We built a prototype of our design by fabricating it on an IC (shown in Fig. 1) and integrating it with a re-programmable antenna on a flexible substrate. The design also integrates a MAC protocol that allows it to scale to multiple sensors. Our evaluation in both in-vitro (fluids) and ex-vivo (tissues) conditions demonstrates the following results:

- $\mu$ medIC's programmable resonance allows it to harvest energy across different types of tissues including fat, muscle, and multilayer compositions with muscle, fat, and bones as well as different fluids. The resonance can be reconfigured by as much as $200 \mathrm{MHz}$ inside tissues. In the absence of reconfigurability, the micro-implant's ability to power up reduces to one or two tissues.

- $\mu$ medIC can support bitrates reliably up to $6 \mathrm{Mbps}$ and as low as $625 \mathrm{kbps}$. Its rate adaptation can gracefully scale to different in-body conditions by incorporating feedback through sensor hints. In the absence of rate adaptation, the design becomes either limited to low availability or low throughput.

Contributions. We present the first batteryless micro-implanted system that is capable of self-reconfiguration for energy harvesting and backscatter communication inside tissues. The system introduces a reconfigurable architecture with programmable antennas, harvesting 
circuits, and backscatter throughput. The design also introduces a rate and resonance adaptation protocol for wireless micro-implants. We also present a prototype implementation on an integrated circuit on a flexible antenna substrate and evaluation in different tissues.

We note that $\mu$ medIC's benefits extend beyond micro-implants that are entirely batteryless. For example, in higher data-rate applications (such as streaming images from endoscope capsules), today about half the energy is spent on RF transmissions [54, 61]. By enabling efficient and reconfigurable backscatter, $\mu$ medIC can significantly reduce the power consumption of such implants, allowing for battery-assisted implementations [44] that can function longer. As the technology evolves, it may also be integrated with recent proposals on battery-free cameras (which have been demonstrated outside the human body) [36]. Such designs are beyond the scope of this paper and are left for future work.

\section{BACKGROUND}

The past two decades have witnessed an increased interest in bringing wireless capabilities to implantable devices. Research in the early 2000's focused on understanding the impact of RF signals on the human body $[28,43]$, and was propelled by the rise of body area networks [13]. The success of this research and technological agenda resulted in wide adoption of wireless communication in implantable medical devices such as implanted pacemakers, cardiac defibrillators, insulin pumps, and capsule endoscopes [20, 61]. These early systems were all battery-powered [13].

The success of this body of work has prompted researchers to extend the vision beyond wireless communication to in-body wireless power transfer $[42,59]$. Power transfer can eliminate the need for batteries which would, in turn, allow implantable sensors to function longer (without surgical replacement) and can result in a significant reduction in their form factor (since batteries can occupy $50 \%$ or more of the sensor's size [15]). These capabilities can significantly expand the potential use cases of in-body sensors to tumor monitoring, neural stimulation, and drug delivery [22, 33, 47, 54]. The promise of such sensors has prompted the US Office of Science and Technology to declare long-lasting wireless micro-implants as one of six grand challenges of the decade [46].

One of the major challenges that still faces in-body wireless applications is the low efficiency of implantable antennas [28, 49]. This low efficiency (around 1\%) has been widely documented in literature on wireless communication with battery-powered medical implants [34, 37], and it becomes even more problematic for batteryless micro-implants that rely on harvested RF energy to power up [33, 54]. Recent advances in energy harvesting try to address this problem by resorting to resonant rectennas, where the antenna and the rectifier (energy harvester) are designed to resonate in order to maximize their harvesting efficiency $[18,24]$. Such resonance, however, is significantly impacted by surrounding tissues; prior work has demonstrated that if tissue composition or depth changes, antennas can easily shift out of resonance, becoming inefficient [30, 32]. This is why the majority of existing in-body sensors still require batteries or remain limited to shallow depths where they can harvest enough energy to power up despite their low efficiency $[53,54]$. Our work is motivated by this past literature on resonant rectennas and extends it to work across tissues by introducing reconfigurability to the design of wireless and batteryless micro-implants.

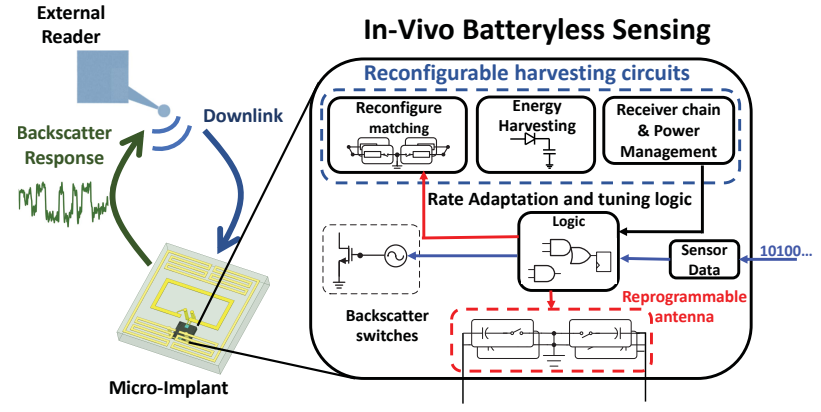

Figure 3- $\mu$ medIC's Design. An external reader powers up the micro-implant which harvests the RF energy, decodes commands, backscatters its response, and adapt its rate and configuration to channel conditions.

\section{SYSTEM OVERVIEW}

$\mu$ medIC is a fully-integrated wireless and batteryless sensor for micro-implants that operate in the UHF (Ultra-High Frequency) ISM Band (902-928 MHz). The sensor can be used to support a variety of in-body monitoring and sensing applications such as tracking biomarkers or long-term monitoring of internal vitals to allow for early intervention.

A $\mu$ medIC sensor powers up by harvesting energy from RF signals transmitted by a reader outside the body. The sensor decodes the reader's downlink commands and transmits its own packets on the uplink to be decoded by the reader. The design extends to multiple sensors, each of which is uniquely addressable. In the presence of multiple sensors, the reader orchestrates medium access.

The overall architecture of a $\mu$ medIC sensor is shown in Fig. 3. The design consists of a system-on-chip (SoC) that supports energy harvesting, decoding, and backscatter communication. $\mu$ medIC's SoC also incorporates a power management unit to support the various computing and communication tasks and an extensible interface that allows integrating the chip with external sensors. The $1 \mathrm{~mm}^{2}$ chip is assembled on a flexible PCB with a custom printed antenna.

$\mu$ medIC can self-reconfigure to adapt to different in-body environments. There are two key components of this self-reconfiguration: the first is a reprogrammable bi-loop antenna that can adapt to surrounding tissues ( $\$ 4)$ and the second is a rate adaptation algorithm that tracks circuit-level sensor hints and adapts to channel conditions (§5). The antenna and rate reprogrammability can be orchestrated by the IC itself. The next sections describe these components in detail.

\section{REPROGRAMMABLE IN-BODY RECTENNA}

In this section, we describe the design of $\mu$ medIC's reprogrammable rectenna and demonstrate how this design enables adapting to different tissues in order to ensure efficient energy harvesting across various in-body environments.

\subsection{Resonant Rectenna Design}

Before delving into $\mu$ medIC's design, it is helpful to understand the challenges that face RF energy harvesting inside tissues and how prior designs address these challenges.

Harvesting RF energy inside human tissues is more challenging than harvesting in air for two main reasons. First, RF signals exponentially attenuate as they traverse human tissues [37, 41], while in air, their amplitude decays linearly with distance. This makes it difficult for an external reader to deliver sufficient energy to power up an 
energy harvesting micro-implant inside tissues. ${ }^{2}$ The second challenge facing in-body energy harvesting arises from the constrained form factor of micro-implants. Specifically, due to anatomical constraints, micro-implants have form factor requirements that vary between 2-3 cm [16] to sub-centimeter dimensions. The limited form factor makes it difficult to efficiently harvest energy since it constrains the dimensions of the micro-implant's antenna with respect to the wavelength of the RF signal [37]. ${ }^{3}$

Because of the above challenges, state-of-the-art proposals for in-body energy harvesting fine-tune their designs to optimize the harvesting efficiency along two main dimensions:

- Radiation efficiency in Bio-tissues: This refers to the efficiency of antennas in transmitting and receiving RF signals within a specific frequency band of interest. Because of the limited antenna form factor and the conductive properties of human tissues, in-body antennas suffer from low radiation efficiencies (they are typically as low as $1 \%$ ) [37]. In order to minimize losses due to the surrounding tissue environments, antenna engineers typically simulate their designs in electromagnetic simulators which account for the impact of the dielectric properties of tissues. This allows them to fine-tune various design parameters (like shape, geometry, thickness of conductor) to achieve the highest possible efficiency given the limited form factor and simulated medium [12].

- Resonance: Aside from optimizing the radiation efficiency of micro-implant antennas, state-of-the-art designs also exploit resonance [25, 45]. Resonance is a well-known electrical property that boosts harvesting energy efficiency by minimizing losses. It can be achieved by electrically matching the antenna impedance to the input impedance of the rectifier (energy harvester).

In order to maintain small form factor and optimize energy harvesting performance, state-of-the-art designs employ electrically small inductive loops, optimize their designs, and match them to the rectifying circuits [26]. The resultant rectennas are most efficient when they operate at their resonance frequency, defined by:

$$
f=\frac{1}{2 \pi \sqrt{L_{A} C_{\text {rect }}}}
$$

where $L_{A}$ is the inductance of the loop antenna and $C_{\text {rect }}$ is the input capacitance of the rectifier.

\subsection{The Impact of Tissues on Resonance}

In our above discussion, we have maintained that it is possible to simulate in-body environments that reflect practical real-world conditions. While that is true in principle, it is very difficult to truly optimize the energy harvesting to reflect practical environments. This is because the human body consists of multi-layer tissues, each layer with a different depth. Moreover, the tissue composition changes across different individuals as well as different body parts or organs. This makes it infeasible to design a one-size-fits-all resonant rectenna that has high efficiency across different body parts (e.g., for mobile micro-implants), let alone for different humans.

The difficulty in adapting to complex tissues arises from differences in their relative permittivity $\epsilon_{r}$ (which reflect differences in the dielectric). Permittivity varies across different tissues, and directly

\footnotetext{
${ }^{2}$ Specifically, state-of-the-art RF rectifiers need around $-34.5 \mathrm{dBm}$ of power to power up [25]. This minimum threshold is determined by transistor electronics.

${ }^{3}$ In the absence of such form factor requirements, achieving good radiation efficiency would require antennas whose dimensions are of the same order of the wavelength [11].
}

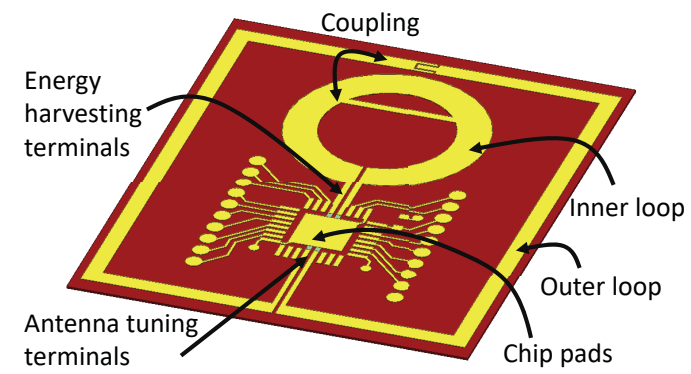

Figure 4-Reconfigurable Antenna. The proposed antenna design showing the inner loop, outer loop, coupling as well as chip ports for energy harvesting and reconfiguration.

impacts the antenna radiation pattern as mentioned earlier. For example, while the permittivity of muscle tissues is $\epsilon_{m}=55-17.4 j$, the permittivity of fat is $\epsilon_{f}=11-2 j$, both around the same frequency of $900 \mathrm{MHz}$ which corresponds to the ISM band of interest [19].

To better understand the impact of such tissue variations on energy harvesting, recall Fig. 2 from the introduction which demonstrated how a design that is optimized to achieve high efficiency in muscle tissue within the ISM band $(910 \mathrm{MHz})$ becomes inefficient at the same frequency when placed in a different tissue (e.g., fat). Indeed, we empirically verify this behavior in real-world measurements in $\S 8$. This behavior demonstrates the difficulty in scaling prior rigid designs to complex tissue environments. Because of this lack of scalability, existing designs are limited to shallow depths, where the received energy remains sufficient to power them up despite their low harvesting efficiency $[53,54] .^{4}$

\subsection{Reconfigurable Coupled Rectenna}

To deal with the above challenges and scale to different in-body environments, $\mu$ medIC adopts a self-reconfigurable architecture that enables it to adapt to surrounding tissues. This reconfigurability is made possible by synergistically combining two sub-components: (1) the first is a reprogrammable antenna that can adapt its radiation efficiency to the surrounding medium, and (2) the second subcomponent is reprogrammable matching circuit that enables shifting the resonance to ensure that the energy harvesting circuit and antenna remain matched. By tuning both the radiation efficiency and the resonance, $\mu$ medIC's design can adapt the two core dimensions that are typically pre-tuned to achieve high harvesting efficiency in tissues as per $\$ 4.1$. The rest of this section describes these two sub-components in detail.

\subsubsection{Antenna Reprogrammability}

To enable antenna reconfigurability, $\mu$ medIC employs a coupled antenna design. Recall that coupling refers to the interaction between two antennas when they are in close physical proximity [11], and is typically considered to be harmful. In our design, however, we exploit a coupled design in order to adapt $\mu$ medIC's radiation efficiency inside tissues. Technically, our goal is to change the current distribution along the radiating element in order to counteract the change in the surrounding medium's permittivity.

Fig. 4 shows $\mu$ medIC's coupled design, which consists of two loops: an outer rectangular loop and an inner circular loop (with a

\footnotetext{
${ }^{4}$ At shallow depths, the RF signals experience less overall (exponential) attenuation due to a shorter path length inside tissues.
} 


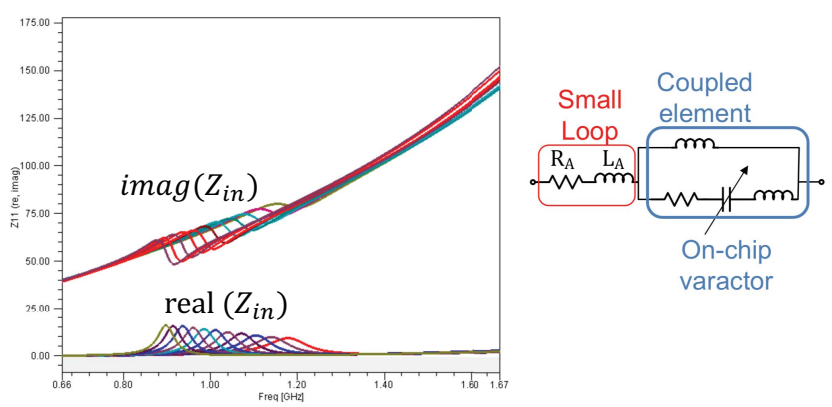

Figure 5-Programmable Antenna Model. The programmable coupled antenna can be modeled as an inductive loop coupled with a resonant LC tank (right figure) whose impedance shows a peak in the real part of its frequency response (left figure).

horizontal chord). Both loops interface with the IC, albeit for different purposes. The inner loop interfaces with the energy harvesting bank of the circuit (i.e., the rectifier) while the outer loop interfaces with the reprogrammability circuit which is implemented as a variable capacitor as we detail below. Because the two loops inductively couple due to their proximity, any change in the radiation pattern of the outer loop gets mirrored in the inner loop, thus changing its radiation pattern and allowing for programmability.

Next, we would like to understand how such a design allows shifting the radiation efficiency across frequencies. A standard approach for understanding an antenna's efficiency is to measure its input impedance. So, we simulated the input impedance of the inner loop and plot the real and imaginary part of that impedance across frequencies in Fig. 5. Each color in the figure represents a different configuration (i.e., a different capacitive load on the outer loop).

One approach to understand where an antenna is efficient is to look at the peak of the real component of its input impedance [11]. For example, right-most green curve has a peak around $900 \mathrm{MHz}$, indicating that the antenna is most efficient around that frequency. However, as we change the load on the outer loop, that peak shifts, indicating that the frequency of highest radiation efficiency also shifts. This shows that $\mu$ medIC's design indeed enables programming the radiation efficiency. Such programmability is highly desirable because if the antenna becomes most efficient around $1.1 \mathrm{GHz}$ due to surrounding tissues, this allows us to shift the highest efficiency region back to the $900 \mathrm{MHz}$ ISM band.

The right side of Fig. 5 shows a conceptual schematic of the resulting coupled design, which is a standard approach to reason about coupled engineering designs [50]. The schematic represents the inductive coupling between the two loops as an inductor, and represents the programmability via an on-chip variable capacitor. In practice, we implemented this programmability as a switched capacitor bank for simplicity and energy efficiency. This schematic would exhibit the same behavior observed in the plots shown to the left as the capacitance is changed.

Finally, we note that this programmable architecture was the result of many iterations in consultation with the relevant literature. Antenna design is an art and is known to require extensive iterations, especially for bio-tissues, and thus it remains an active area of research [12]. One of the interesting designs we explored consisted of a single loop, where one side of the loop wass connected to energy harvesting while the other was connected to a programmable load;

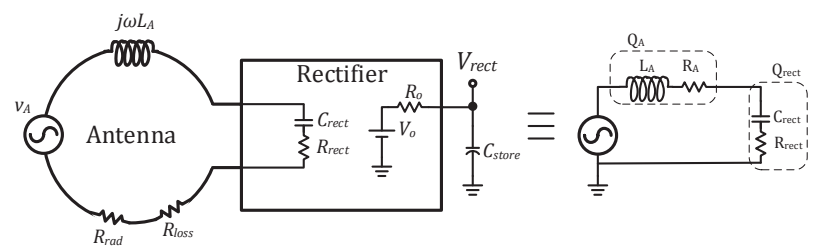

Figure 6-Rectenna Circuit Schematic. The rectenna can be modeled as a resonant LC circuit where the inductance is contributed by the antenna and the capacitance represents the rectifier impedance.

such a design, however, had a much lower bandwidth than $\mu$ medIC's coupled architecture which resulted in significantly lower data rates. ${ }^{5}$ Another interesting design element is the horizontal chord across the inner loop, which has also been recently demonstrated to achieve wider bandwidth and higher efficiency (albeit without reprogrammability) $[25,45]$. Thus, because antenna design is a highly complex task, by introducing a simple design that enables reprogrammability, $\mu$ medIC can reduce the burden on pre-tuning all the parameters and allow for reconfigurability to adapt to different media.

\subsubsection{Harvesting Reconfigurability}

So far, we have focused only on improving the antenna's efficiency by shifting its resonance frequency. However, if we just do that, then we cannot ensure it remains matched to the energy harvesting circuit. Thus, we won't reap the benefit of resonance harvesting.

To optimize the energy harvesting efficiency, rectennas rely on the perfect resonance between an electrically small antenna with an inductive impedance profile with the conjugately matched capacitive input impedance profile of the rectifier circuit as shown in Fig. 6. This can be expressed in the following equation:

$$
Z_{A}=Z_{\text {rect }}^{*}
$$

where $Z_{A}$ is the antenna's impedance while $Z_{\text {rect }}$ is the rectifier's input impedance.

Now recall that in Fig. 5, the antenna programmability changes $Z_{A}$. So, we should change the rectifier accordingly to ensure resonance occurs at all programmable states.

To achieve this, we introduce programmable circuit matching. Since the programmable antenna is still an inductive one and the rectifier has a capacitive impedance, the required matching network can be implemented as a simple bank of capacitors. This capacitor bank can be used to compensate for the change in inductance from one state to the other, thus maintaining perfect conjugate matching. Effectively, by reprogramming both the antenna and the harvesting circuit, we have disposed of the need to pre-fine-tune the design and allowed ourselves to retune it inside the human body.

One might wonder whether modifying the antenna alone or the energy harvesting alone might be enough. Implementing any of these approaches alone will lead to less desirable performance. Changing the matching alone can allow a slight shift in the center frequency (e.g., a slight shift in the channel) [51], but if the desired shift is too large, the antenna becomes inefficient. On the other hand, reprogramming the antenna alone would not be enough because it would significantly reduce the efficiency without resonance. It is by

\footnotetext{
${ }^{5}$ Indeed, coupled designs are known to have larger bandwidth, and hence have been used in the design of wideband RFIDs and UWB front-ends [3, 17]. In contrast to prior coupled designs which are rigid, $\mu$ medIC's design allows for reconfigurability.
} 
combining both of these techniques together that $\mu$ medICcan enable cross-tissue wireless and batteryless connectivity for micro-implants.

\section{RATE ADAPTATION FOR IN-BODY BACKSCATTER}

So far, our discussion has focused on $\mu$ medIC's energy harvesting reconfigurability. Next, we discuss how it can adapt its bitrate to deal with varying channel conditions. Specifically, recall that $\mu$ medIC communicates on the uplink via backscatter. Moreover, it needs to support a variety of applications, some of which may require bitrates up to few Mbps (e.g., capsule endoscopes) [15]. Thus, it is desirable to enable the sensor to backscatter at the highest possible bitrate needed by the application of interest whenever possible. Below, we discuss the need for in-body backscatter bitrate adaptation and how $\mu$ medIC's reconfigurable design can address these needs.

\subsection{The Need for Adaptation}

Bitrate adaptation is today a core component of a variety of wireless network protocols, including WiFi and LTE. It refers to the ability of certain communication devices to adapt their throughput to the channel conditions. For example, if the channel is strong (i.e., has high SNR), the communication link can sustain higher throughputs and the transmitter should use a higher bitrate since the channel capacity is higher. On the other hand, if the channel is weak (i.e., low SNR), the transmitter should transmit at a lower bitrate.

Bringing such bitrate adaptation to in-body micro-implants is desirable for two main reasons. First, as the person moves (or as the micro-implant moves inside the body), the wireless channel changes and the bitrate must adapt to it. A second, and equally important, reason arises from the relationship between power consumption and backscatter bitrate. Specifically, higher bitrates consume more energy because the oscillator needs to be driven at a higher frequency. ${ }^{6}$ (The overall power consumption $P$ is directly proportional to bitrate $f$ according to $P=f C V^{2}$ where $C$ is the capacitance and $V$ is the voltage.) Thus, the bitrate also needs to be adapted to the harvested energy to ensure that the micro-implant does not consume all its energy and die off.

To gain more insight into the trade-off between bitrate and power consumption, we ran experimental trials with $\mu$ medIC (implementation detailed in §6). In each experimental trial, we pre-programmed the micro-implant to backscatter at a fixed bitrate. We repeated the experiment at three different bitrates (600kbps, 3Mbps, 6Mbps) and two different transmitted power levels $(14 \mathrm{dBm}$ and $15 \mathrm{dBm})$. We specifically selected two power levels that are close to demonstrate that $\mu$ medICneeds to adapt to changes as small as $1 \mathrm{~dB}$. In each experimental trial, the external reader is programmed to alternate between transmitting for $200 \mu$ s and pausing (i.e., not transmitting anything) for $200 \mu \mathrm{s}$. In each trial, we measured $\mu$ medIC's harvested DC voltage and the internal ring oscillator's clock voltage over time.

Fig. 7 plots the measured voltages across the three different fixed bitrates and the two power levels.

We make the following remarks:

- The harvested voltage in Fig. 7(a)-(c) at the higher power is larger when the transmitted power by the external reader is $15 \mathrm{dBm}$ (red

\footnotetext{
${ }^{6}$ Note that this bitrate frequency of the backscatter oscillator is different from the RF frequency of transmission.
}

plot) than when the transmitted power is $14 \mathrm{dBm}$ (blue plot). This is expected since higher power leads to more harvested energy.

- In the high-transmit-power low-rate regime (Fig. 7(d)), $\mu$ medIC is able to continuously backscatter for the entire duration that the external reader is on; in fact, the node has enough energy to backscatter for a longer duration, even after the external reader is turned off (due to the excess harvested energy). Moreover, even in the lower power regime (Fig. 7(g)), it can backscatter almost for the entire duration of the external reader's transmission.

- At the other extreme, when $\mu$ medIC is programmed to the higher rate of $6 \mathrm{Mbps}$, it can only power up at the higher reader power, and even then, it only transmits continuously for $20-50 \mu$ s before the oscillator dies off to recharge again, causing the duty-cycled operation shown in Fig.7(f). When the reader's power is lower, it can't power up or oscillate steadily as depicted in Fig. 7(i).

- Finally, the medium rate of $3 \mathrm{Mbps}$ sits at a sweet spot where it can achieve higher overall throughput while maintaining the ability to power and continuously transmit without frequently dying off during backscatter.

In principle, one could preprogram the micro-implant to conservatively backscatter at the lowest bitrate. However, this would not be desirable as it would forgo the opportunity to enable higher throughput. In the next section, we describe how $\mu$ medIC can adapt its rate to the available energy.

\subsection{Throughput Programmability}

In order to take advantage of good channels, and at the same time, not die off at low channels, $\mu$ medIC performs bitrate adaptation. The standard approach for bitrate adaptation is to wait for the receiver to transmit acknowledgment packets. However, such approach is undesirable for multiple reasons. First, we want to adapt the bitrate before the sensor dies off. Second, the overhead for waiting for an acknowledgment results in unnecessary loss of harvested energy [55].

To close the loop on bitrate adaptation, $\mu$ medIC monitors circuit level sensor hints. Specifically, it senses the harvested voltage and preemptively adapts its bitrate before dying.

Next, we describe how this can be realized in practice. $\mu$ medIC's backscatter switch is driven by a digitally controlled oscillator. The oscillator's frequency can be in one of eight different states (i.e., 3 control bits). By employing a current-starved ring oscillator architecture, we can digitally tune its frequency by injecting more current to the ring oscillator in order to increase the frequency or by starving the core ring so that a lower frequency can be attained. The rectenna tuning logic senses the DC voltage and incrementally increases or decreases the oscillator frequency. This frequency is used to modulate and encode the sensor bits and backscatter to the reader. Alg. 5.1 summarizes this algorithm, where $V_{D C}, V_{T H}$, and $D_{\text {fosc }}$ represent the harvested voltage, turn-on threshold, and oscillator's frequency configuration respectively.

Few additional points are worth noting:

- So far, we have described $\mu$ medIC's bitrate adaptation algorithm. The same idea can be extended to resonance adaptation as shown in Fig. 8. Specifically, by sensing the harvested voltage using a sampler (ADC), the node can apply stochastic gradient descent to move to a more efficient harvesting state by updating the matching circuit and resonance capacitance. It is worth noting, however, that unlike rate adaptation, resonance and matching adaptation 


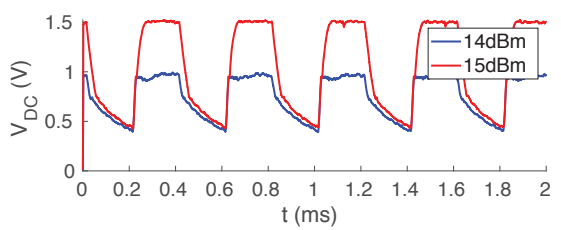

(a) Harvested voltage for low throughput

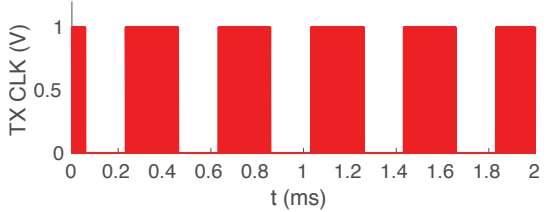

(d) Transmitter clock at $15 \mathrm{dBm}$ and $600 \mathrm{kbps}$

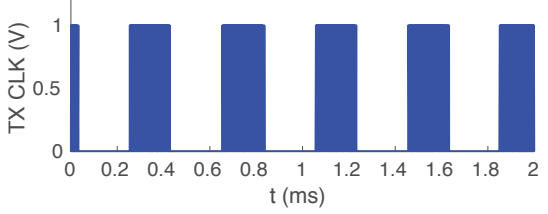

(g) Transmitter clock at $14 \mathrm{dBm}$ and $600 \mathrm{kbps}$

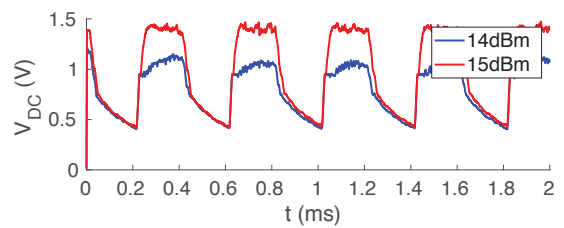

(b) Harvested voltage for medium throughput

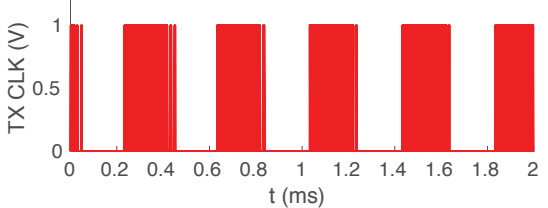

(e) Transmitter clock at $15 \mathrm{dBm}$ and $3 \mathrm{Mbps}$

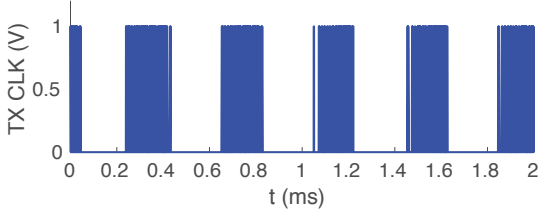

(h) Transmitter clock at $14 \mathrm{dBm}$ and $3 \mathrm{Mbps}$

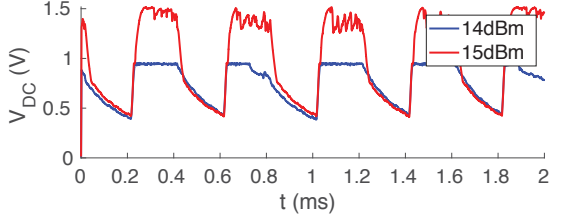

(c) Harvested voltage for high throughput

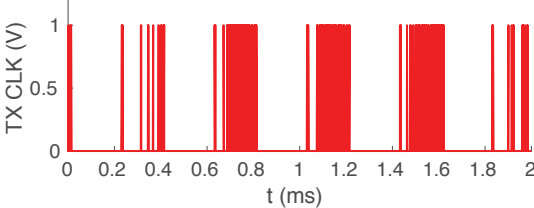

(f) Transmitter clock at $15 \mathrm{dBm}$ and $6 \mathrm{Mbbps}$

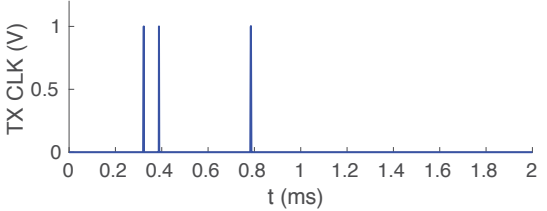

(i)Transmitter clock at $14 \mathrm{dBm}$ and $6 \mathrm{Mbps}$

Figure 7-Availability vs Bitrate and Power Level. The figure plots the availability of $\mu$ medIC's communication as a function of different bitrates and power levels. The different columns represent different throughputs in increasing order from left to right (600kbps, 3Mbps, $6 \mathrm{Mbps}$ ); the colors represent different transmit powers (blue for $14 \mathrm{dBm}$ and red for $15 \mathrm{dBm})$. The top row represents the voltage harvested over time for the two power levels. The middle and bottom row show the periods in time with the sensor is on (1V) and off $(0 \mathrm{~V})$.
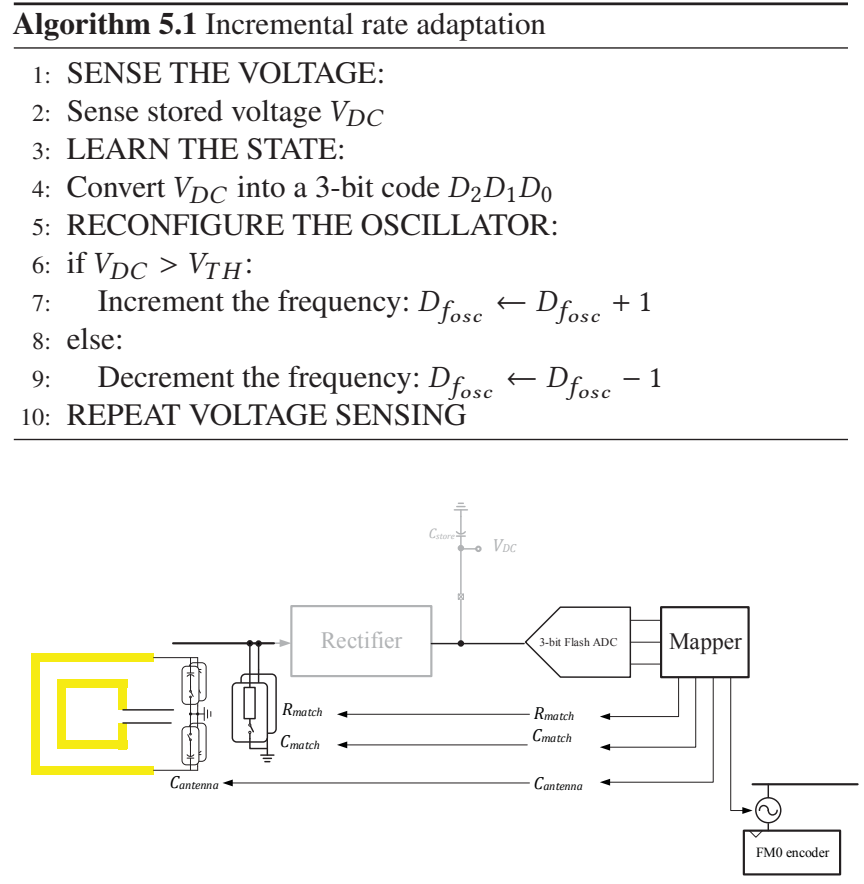

Figure 8-Self-Reconfiguration Logic. $\mu$ medICsamples the stored DC voltage and maps it to discrete values for the antenna configuration, matching state, and bitrate.

require the off-chip storage super-capacitor to have some residual energy in the first place. This "warm-start" approach is a standard assumption in the design of energy harvesters [45]. Extending the design to cold-start is beyond the scope of this paper.

- There are three types of reconfiguration parameters (matching capacitor bank, resonance capacitor bank, and oscillator frequency). Each of these parameters can be reconfigured independently. The two capacitor banks can be adapted on a packet-by-packet basis
In the simplest implementation, we alternate between incrementing/decrementing each of the two capacitor banks. Looking ahead, it would be interesting to explore more optimal algorithms that can leverage $\mu$ medIC's reconfigurable design to achieve higher throughput than our simple adaptation algorithms.

- Our discussion has focused on rate and resonance adaptation. However, $\mu$ medIC's design extends to decoding packets on the downlink (via PIE encoding) and encoding backscattered packets on the uplink via FM0 encoding similar to RFID communication. The ability to decode downlink packet enables the reader to employ a master-slave Medium Access Control (MAC) protocol and extend to multiple micro-implanted sensors concurrently.

- In our evaluation, we noticed that the backscatter bitrate varied over time, even when the oscillator was expected to transmit at a fixed rate. This was caused by the susceptibility of the node's ring oscillator to environmental variables. To deal with this issue and decode correctly, our reader continuously tracks the backscatter frequency and adapts to its variations. Practically, this was implemented via a bit-by-bit maximum likelihood decoder that corrects phase/frequency errors incrementally from one bit to the other.

In sum, the rate adaptation operates on a packet-by-packet basis where the chip senses the voltage and increments or decrements the bitrate accordingly. On the receive side, the reader uses the preamble of the backscattered packet in order to determine the backscatter rate and correctly decode the sensor's data.

\section{IC DESIGN \& ANTENNA FABRICATION}

ASIC Implementation. $\mu$ medIC is implemented as an applicationspecific integrated circuit (ASIC), whose prototype is shown in Fig. 1. The integrated circuit was designed and fabricated in a $65 \mathrm{~nm}$ low-power RF complementary metal-oxide-semiconductor (CMOS) technology. The ASIC incorporates the entire system-on-a-chip, and 


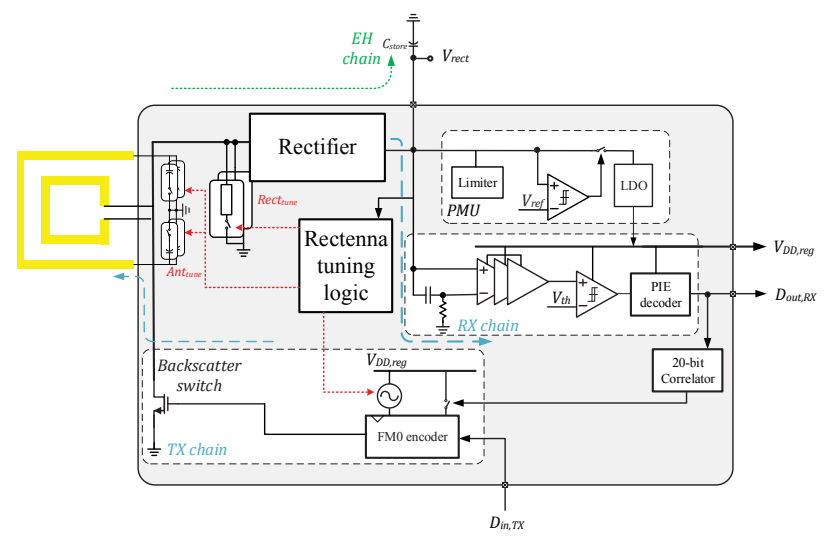

Figure 9- $\mu$ medIC's Chip Block Diagram. The chip consists of energy harvesting and power management building blocks as well as receive and transmit chains. The tuning logic controls the chip's configuration as well as the system's state of operation.

integrates all the antenna and circuit programmability, logic, and communication functionality onto a single $1 \mathrm{~mm}^{2}$ chip.

Coupled Antenna on Flexible Substrate. The ASIC is wirebonded to the coupled antenna structure on a printed antenna on a flexible PCB. The programmable coupled antenna consists of two microstrip copper loops. The two coupled loops enable reconfiguration of the antenna centered around ISM band (902 - 928MHz).The flexible PCBs were simulated in High-Frequency Structure Simulator (HFSS) [2] and fabricated by FlexPCB [5]. The chip and the antenna flexible board are encapsulated in a biocompatible polymer (Ecoflex polymer) to mitigate any reaction with the human tissues and insulate the node from the harsh in-body environment. Note that while our final evaluation was performed with flexible substrate, we have also successfully tested the ASIC with different antenna designs and on rigid substrates including FR-4. The overall antenna design went through multiple iterations where we compared the impedance in simulation to that measured using a network analyzer.

Overall Architecture and Energy Harvesting. The integrated system block diagram is depicted in Fig. 9 which consists of the rectifier, the power management unit (PMU), the rectenna tuning logic, the receiver chain, the transmitter chain, and other auxiliary blocks. The energy harvesting chain incorporates a 6-stage differential rectifier with a self- $V_{t h}$ cancellation technique to generate a constant voltage over an off-chip storage capacitor using the CW RF input signal. The role of the power management unit (PMU) is to limit and regulate the harvested voltage in order to provide a stable supply for the more sensitive communication circuits and logic. The PMU was implemented using a voltage limiter and a low-dropout regulator (LDO) to provide regulated power supply to the rest of the circuit.

Receive Chain. The input modulated signal follows the receiver chain where it gets amplified, filtered and demodulated. Then, it passes through a decoder which converts the Pulse Interval Encoded (PIE) symbols into output bits. Finally, a 20-bit correlator correlates the received packet MAC address to a stored 20-bit sequence to determine whether this packet is addressed to it or to another implant.

Transmit Chain. On the uplink side, once a full correlation is achieved, the regulated supply turns on a tunable ring oscillator which generates the clock signal for an FM0 encoder and a data sampler to provide the modulating signal for the backscattering

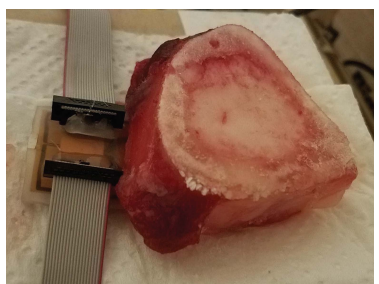

(a) Mixed tissue

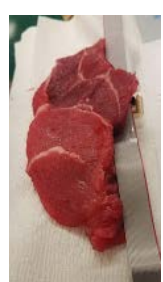

(b) Lean tissue

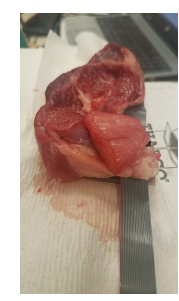

(c) Fatty tissue
Figure 10-Test setups for $\mu$ medIC. The figures shows test setups in: (a) a mixed tissue, (b) a lean meat tissue, and (c) a fatty tissue.

switch, completing the whole transmitter chain. We implemented the oscillator as a digitally controlled 6-bit current-starved ring oscillator which is used to control and gracefully adapt the backscatter rate of the micro-implant. The oscillator can sustain up to $20 \mathrm{Mbps}$ throughput; however, the rate adaptation limits it to $6 \mathrm{Mbps}$.

Reconfigurability. For the rectenna reconfigurability logic, we implemented dual capacitor tuning for resonance as well as harvesting reprogrammability. The chip utilizes two 6-bit capacitor banks, each of which has 64 discrete capacitor values. Both capacitor banks are implemented as binary weighted capacitors connected to the inner or outer loops of the antenna through individual selection switches. For the antenna resonance tuning, the capacitor bank is connected to the differential load terminal of the coupled antenna. For the rectifier harvesting tuning, the capacitor bank is connected in parallel with the rectifier in order to tune the input impedance of the chip.

\section{EVALUATION}

We evaluated $\mu$ medIC across five kinds of in-body environments including: minced meat, fatty tissues, mixed tissues with bone, saline water, and oil-based fluids. Since the majority of human tissues are oil-based or water-based, this allows us to cover a large variety of potential environments and multi-layer tissues. In each of these environments, we either submerged the sensor entirely in the fluid or covered the antenna with meat tissues as in Fig. 10. In addition to the different tissues, we evaluated our setup at different transmit powers, orientations, as well as distances ranging from $10 \mathrm{~cm}$ (between the reader and the tissue with $\mu$ medIC) at low power levels to almost a meter at a transmit power of $20 \mathrm{dBm}$. The pins of the IC are connected via a $1.27 \mathrm{~mm}$ pitch bus to a Tektronix MSO3054 oscilloscope and to an FPGA to allow for testing and voltage measurements. Note that our design allows bypassing the self-reconfigurability to allow for external configurability and testing each state independently. Moreover, an Agilent N9020A spectrum analyzer [1], hooked directly to an antenna, was used to initially characterize the backscatter modulation depth at different rates and center frequencies.

Subsequently, our complete end-to-end evaluation was performed using a software radio setup which employed two USRP N210 [10] boards for collecting and post-processing a large number of packets and estimating the BER under different channel conditions. One USRP board (with an SBX daughterboard [9]) served as the transmitter for the micro-implant, powering the chip up and providing downlink commands. The other USRP (with LFRX daughterboard [7]) worked as the reader, capturing the backscatter signal at a sampling rate of $25 \mathrm{MSps}$. Both USRP boards were synced together using the same reference clock of $10 \mathrm{MHz}$ of a CDA-2990 Octoclock 8channel distribution module [4]. Moreover, their front-ends were 
connected to log-periodic antennas [8] and their backends were connected via ethernet cables to allow for a central Linux machine to control both of them simultaneously. The data was then collected and post-processed using MATLAB to characterize the bit error rate, SNR, throughput and availability in the different tissues under test.

\section{PERFORMANCE RESULTS}

In this section, we report the evaluation results of $\mu$ medIC's performance in real-world environments and various in-tissue conditions.

\subsection{Energy Harvesting}

First, we evaluated $\mu$ medIC's ability to harvest energy across a variety of in-tissue environments. To do this, we used the Keysight N5183 MXG signal generator [6] as an RF source with fixed transmitted power. The signal generator was connected to a wideband log-periodic antenna covering the range of interest. We swept the transmitted frequency and recorded the received signal using an oscilloscope hooked up to the storage capacitor pin of $\mu$ medIC.

Fig. 11(a) shows the harvested voltage of a rigid design, i.e., when the tuning capacitor (of the antenna) and the matching capacitor are fixed. The figure plots the harvested voltage as a function of the frequency of the signal transmitted by the reader. We repeated the same measurement across the five tissue environments described in $\$ 7$ with testing depths of around $10 \mathrm{~cm}$ or less. The figure also plots a solid black line around $0.65 \mathrm{~V}$, which indicates the minimum voltage required to turn on the low drop-out voltage regulator (LDO). The figure shows that for this fixed configuration, the micro-implant can be powered up in the ISM band only when it is placed in the oil-based tissue (red curve), where the harvested voltage is above the minimum threshold. For all other tissues, the peak is either shifted outside the ISM band (shaded grey region), or the rigid design is completely unable to power up due to low efficiency.

Next, we were interested in assessing whether $\mu$ medIC's reconfigurability allows it to power up in the ISM band. So, for each tissue environment, we looped through all 4096 configurations (changing the tuning and matching capacitors). For each tissue, we chose the configuration that has the highest harvesting voltage in the UHF ISM band. Fig. 11(b) plots the resulting curves. The figure shows that across all tissues, $\mu$ medIC can harvest enough energy inside the UHF ISM band to power up (the harvested voltage is at or above the threshold). It also shows that the optimal configuration for energy harvesting is different across tissues. For example, optimal powering up in saline fluid can be achieved at with a matching capacitor $C_{m}=0.5 p F$ and a tuning capacitor $C_{t}=0.5 p F$; however, optimal powering in mixed tissue requires $C_{m}=0.75 p F$ and $C_{t}=0 p F$. This demonstrates that both the antenna programmability and matching programmability are necessary to adapt to different tissues.

Next, we were interested in understanding the extent of reconfigurability of $\mu$ medIC's harvesting efficiency. This time, we fixed the tissue to an oil-based medium, and we looped again through all the configurations. For each frequency between $750 \mathrm{MHz}$ and $1050 \mathrm{MHz}$, we chose the configuration that yielded the highest harvested voltage. Fig. 11(c) plots the envelope of these peaks across frequencies (in purple) as well as a sample subset of configurations. The figure shows that the peak indeed moves across different configurations by up to $200 \mathrm{MHz}$. Such range of frequency shift is desirable as it allows us to also exploit other bands. For example, the $\mathrm{CCC}$ band in China may also be used for UHF communication and

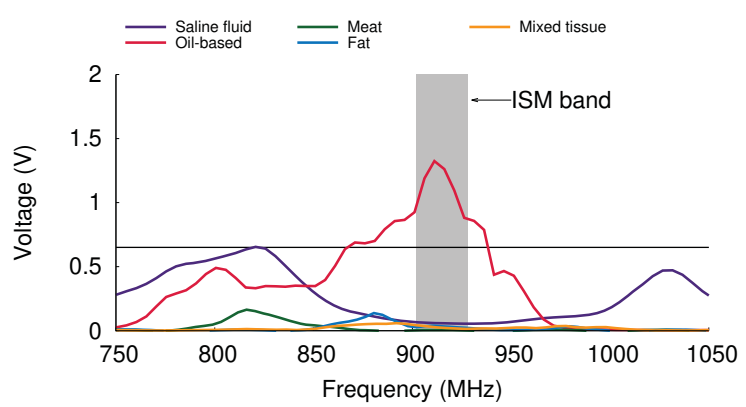

(a) Single Rigid Configuration

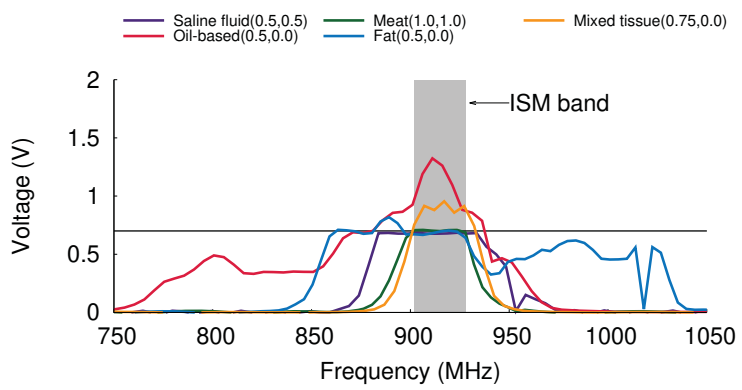

(b) Optimal Configurations in ISM band

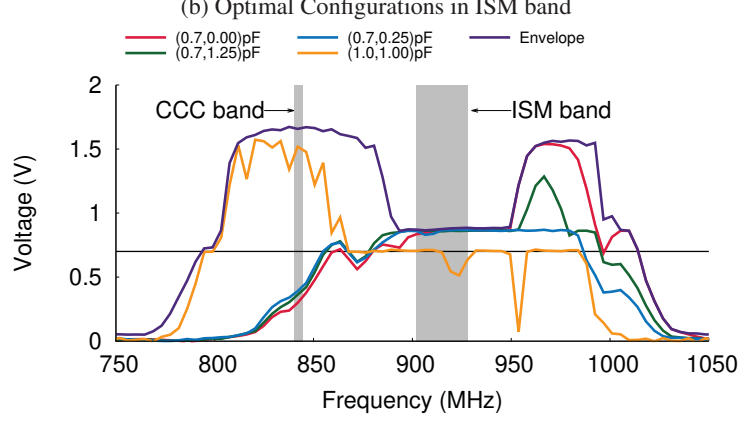

(c) Envelope of Configurations in Oil-based

Figure 11-Energy Harvesting in Tissues. The figure plots the harvested DC voltage as a function of frequency across: (a) different tissues for a single rigid chip configuration, (b) different tissues while programming for the optimal configuration in the ISM band, (c) a fixed oil-based tissue while choosing the highest energy harvesting configuration to cover the widest bandwidth (shown by the envelope contour in purple).

is also shaded in the figure. Thus, $\mu$ medIC's reconfigurability would also allow adapting to regulating bodies across countries.

Finally, we were interested in investigating whether deforming the antenna impacts is resonance (e.g., rolling it as a pill). Our experimental evaluation verified that such deformation may indeed shift the energy harvesting peak by up to $60 \mathrm{MHz}$. Moreover, similar to our earlier demonstration, $\mu$ medIC's reconfigurability enables shifting the resonance back to within the ISM band.

\subsection{Rate adaptation}

Next, we wanted to assess the second dimension of reconfigurability in $\mu$ medIC's design, namely its bitrate adaptation. Recall that bitrate adaptation is necessary to allow adapting to different channel conditions as well as harvested power levels, as highlighted in $§ 5$.

(a) Power Consumption vs Throughput. First, we were interested in measuring $\mu$ medIC's power consumption as well as the impact of throughput on it. To perform this evaluation, we connected the IC to an external power supply of $0.5 \mathrm{~V}$ rather than to its internal LDO and we measured the current drawn using the Keithly 2400 


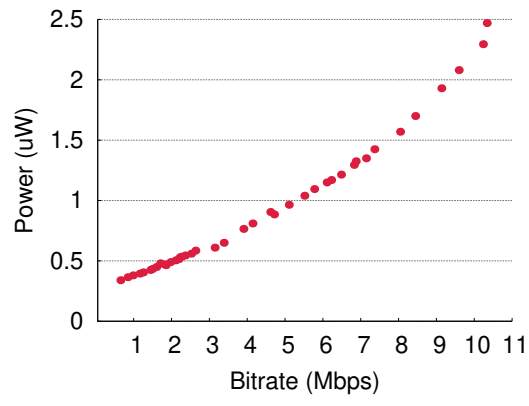

Figure 12-Power Consumption vs Bitrate. The figure plots the average power consumption against the bitrate, showing that higher bitrates consume more power.

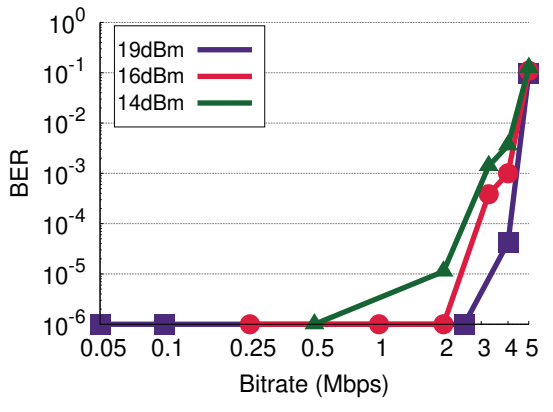

Figure 13-BER vs Bitrate. The figure plots the bit error rate as a function of bitrate at different power levels: $14 \mathrm{dBm}$ (green), 16dBm (red), and 19dBm (purple).

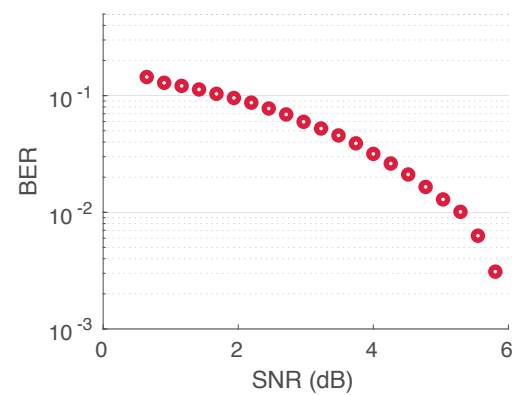

Figure $14-$ BER vs SNR. This figure plots the bit error rate against different SNR levels showing that it follows the expected theoretical trend. sourcemeter [27]. We repeated the same measurement at different clock frequencies (i.e., throughput).

Fig. 12 plots the average power consumption against the transmitter bitrate where the lowest configuration oscillates at a rate of $600 \mathrm{kHz}$ while consuming power as low as $340 \mathrm{nW}$. On the other end, when the available input RF power is high, the node can trade-off its power consumption for a higher bitrate up to $10 \mathrm{Mbps}$ at the expense of $2.5 \mu \mathrm{W}$ of power. Therefore, with an adaptive scheme, the node can scale its datarate as well as power consumption by an order of magnitude according to the available power.

(b) BER-Throughput Curves. Next, we were interested in assessing the importance of bitrate adaptation to deal with varying channel conditions. We ran 90 experimental trials in total. In each experimental trial, we fixed the backscatter bitrate and the transmit power (of the external reader), and we transmitted for a fixed duration of $500 \mathrm{~ms}$ of packets of 128 bits each. At each bitrate and power level, we ran 3 experimental trials, and we experimented with 10 bitrates in total (50kbps, $100 \mathrm{kbps}, 250 \mathrm{kbps}$, 500kbps, $1 \mathrm{Mbps}, 2 \mathrm{Mbps}, 2.5 \mathrm{Mbps}$, $3 \mathrm{Mbps}, 4 \mathrm{Mbps}, 5 \mathrm{Mbps}$ ) and three transmit power levels to correspond to different in-tissue conditions (19dBm, 16dBm, 14dBm). We computed the BER for each bitrate and transmit power as the fraction of incorrectly decoded bits to the total bits transmitted. Recall that the backscatter encoder uses FM0, and our external reader uses a maximum likelihood decoder.

Fig. 13 plots the BER as a function of backscatter bitrate for each of the three power levels (purple for $19 \mathrm{dBm}$, red for $16 \mathrm{dBm}$, and green for $14 \mathrm{dBm}$ ). The figure shows that as the bitrate increases, the BER also increases. This is because higher bitrate requires larger bandwidth, thus increasing the received noise. This figure also shows that if the transmit power increases, the BER decreases; this is also expected because a higher transmit power results in higher backscatter (reflected) SNR, resulting in better decoding ability. This demonstrates the need to adapt the channel not only to the harvested energy as described in $\S 5$ but also to the SNR.

(c) BER-SNR Curves. Recall that the external receiver is implemented as a maximum likelihood detector. Next, we evaluated the receiver's ability to correctly decode backscatter packets transmitted on the uplink. This is typically assessed by plotting the BER-SNR curve. Fig. 14 plots the BER-SNR of the receiver from the same experimental trials described above. It shows that the BER decreases with increased SNR following the expected theoretical trend. (d) Assessing Adaptation. Our final evaluation focuses on understanding the trade-off between availability and throughput and how $\mu$ medIC's bitrate adaptation algorithm (described in \$5) allows adapting the bitrate without sacrificing availability. To this end, we compare the performance of three different schemes, all in timevarying channels. The first uses a fixed low backscatter rate of around $625 \mathrm{kbps}$; the second uses a fixed high rate of around $10 \mathrm{Mbps}$; the third adapts its rate according to the energy stored in the harvester's storage capacitor as per the algorithm described in 5 .

Fig. 15 shows the three different schemes in time-varying channels. The top row plots the harvested voltage versus time for each of the three schemes. The middle row plots the spectrogram (representing the backscatter frequency)which is shown as a heatmap where high power at a specific frequency is indicated by red or yellow and where blue indicates the absence of the corresponding frequency. And finally, the bottom row shows the instantaneous bitrate inferred from the spectrogram. Figs. 15(a), (b), and (c) represent the lowbitrate, high-bitrate, and adaptive bitrate schemes respectively.

We make the following remarks:

- For the low-bitrate scheme, throughput remains constant at around $625 \mathrm{kbps}$ without taking advantage of higher available power at better channels. This can be seen both from the spectrogram and the instantaneous bitrate. The oscillator rarely dies in this scheme due the low power consumption and conservative nature.

- When the node fixes its rate to the high mode regardless of the available power, it suffers from frequent power downs during transmission as illustrated in the spectrogram of Fig. 15(b).

- Finally, the adaptive scheme shown in Fig. 15(c) can smoothly adapt its bitrate from one packet to the other according to the received power variations.

To gain more insight into the tradeoff between availability and throughput, we collected measurements across three schemes, similar to the ones described above: a fixed low rate of $750 \mathrm{kbps}$, a fixed high rate of $12 \mathrm{Mbps}$, and an adaptive rate scheme capable of varying between the two. We ran around 30 experimental trials in total covering low-power, high-power, and varying-power environmental setups. In order to ensure the nodes experienced a time-varying channel in the latter, we programmed our downlink transmitter to quickly vary its transmit power by a factor of 2 each $20 \mathrm{~ms}$. In each experimental trial, we estimated the node failure probability as our availability metric. The empirical probability was computed as the ratio of period of time the node fails (due to running out of power) to the full duration of the transmission. 

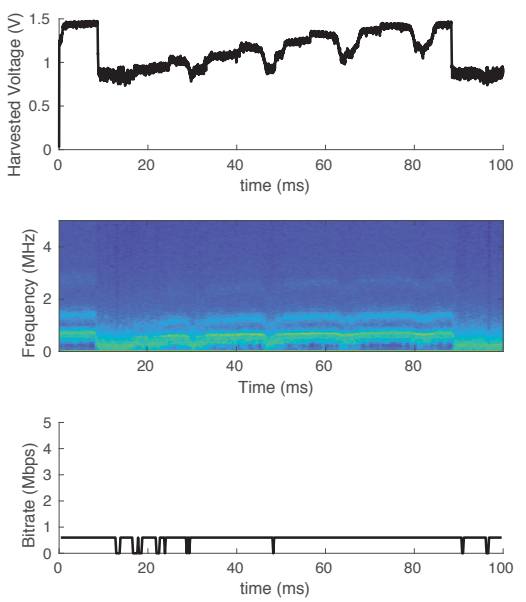

(a) Fixed Low-Rate Configuration
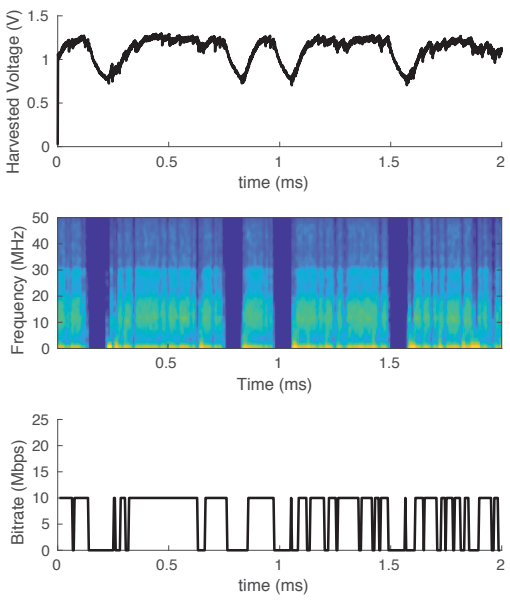

(b) Fixed High-Rate Configuration
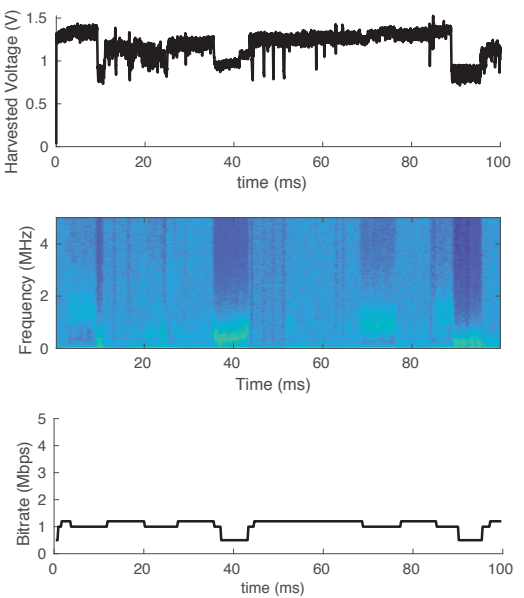

(c) Adaptive-Rate Configuration

Figure 15- $\mu$ medIC across Fixed and Adaptive Schemes. The figure plots $\mu$ medIC's performance across different configurations: (a) fixed low-rate, (b) fixed high-rate, and (c) adaptive rate. The top row plots the harvested DC voltage over time. The middle row plots the oscillator frequency as a spectrogram heatmap over time, where yellow indicates high-intensity and blue indicates low-intensity. The bottom row plots the effective bitrate across time.

Fig. 16 shows the results of this experiment by plotting the node failure probability across the different schemes and setups, where Fig. 16(a), (b), and (c) represent the low-power, high-power, and varying-power setups respectively. The bar graphs correspond to the median failure probability and the error bars show the standard deviation. We make the following remarks:

- $\mu$ medIC's low-rate scheme provides the highest availability. Specifically, even under the lowest-power mode, it's failure probability remains below 0.06 (i.e. $6 \%$ ), and it drops further to 0.0055 at higher power channels. This is expected since the low-rate scheme consumes the lowest power (as per Fig. 12).

- The high-rate scheme suffers from a node failure probability of 0.2 in the low-power regime. This demonstrates that high-rate is likely to be unsuitable for applications that require always-on availability for in-body sensing (and communication).

- In the high-power setup (Fig. 16(b)), almost all modes have high availability, with even the high-rate mode having a failure probability lower than 0.0003 . This suggests that in high-power settings, there is little to be gained from using the low-rate scheme.

- Finally, $\mu$ medIC's adaptive scheme offers a middle ground between low-rate and high-rate. In principle, it is possible to make the adaptive rate more conservative or aggressive by adjusting the adaptation thresholds, which would result in performance that is closer to the low-rate or high-rate schemes respectively.

So far, we have demonstrated that $\mu$ medIC's ability to choose and/or adapt its bitrate enables it to adapt for availability. Next, we would like to understand how these different schemes compare in terms of effective throughput. To do this, we used the same experimental trials described above and performed trace-driven simulations to measure effective bitrate. We also compared different packet sizes to understand the impact of packet size on throughput. The effective bitrate is computed as the number of total packets successfully transmitted (before failing) multiplied by the number of payload bits per packet and divided by the entire transmit duration. Note that if a node fails in the middle of a packet transmission, we consider the entire packet to be lost, thus emulating practical flows.
Fig. 17 plots the effective bitrate for each chip configuration across the different schemes and setups, where Fig. 17(a), (b), and (c) represent the low-power, high-power, and varying-power setups respectively. The figure also shows the effective bitrate for different packet sizes, 100-bit packet (shown in blue), 1000-bit packet (shown in orange), and 5000-bit packet (shown in yellow). The bar plot represents the median rate while the error bar represents the standard deviation. We make the following remarks:

- The high-rate scheme provides the highest throughput (effective bitrate) across almost all setups, even the low-power setup. In particular, for the 100-bit packet size, it achieves bitrates between $8.5 \mathrm{Mbps}$ and $11.5 \mathrm{Mbps}$ across the different setups. While this may be counter-intuitive (given that the high-rate has the lowest availability as per Fig. 16), it is able to transmit more bits within the same duration of time due to its higher bitrate. This shows that if the goal is to achieve the highest throughput (rather than the highest availability), then it is more desirable to configure $\mu$ medIC to its high-rate mode.

- The 100-bit packet size (blue) outperforms all other schemes, despite that it suffers from more overhead since it has the same header size as the other schemes but smaller payload. This is because the chip is prone to suffer from node failure; thus, it is more desirable to get a full packet across than it is to aim for transmitting a longer packet with less overhead.

- Interestingly, the adaptive rate mode performs the best for largest packet size (5000-bits) in time-varying channel, i.e., in Fig. 17(c). This is because it can transmit at higher bitrates than the low-rate and does not fail as frequently as the high-rate configuration.

These results demonstrate that $\mu$ medIC's ability to switch between different configurations (whether fixed or adaptive bitrate) allows reconfiguring it to the application requirements. For instance, a streaming application may require continuous transmission, and thus favor availability over throughput (i.e., low-rate). Other applications may require higher throughput (e.g., taking one snapshot image and transmitting it quickly, with little buffering), and such applications may choose either high rate or aggressive rate adaptation. More generally, these results demonstrates the value of $\mu$ medIC's programmable throughput to serve different application requirements. 


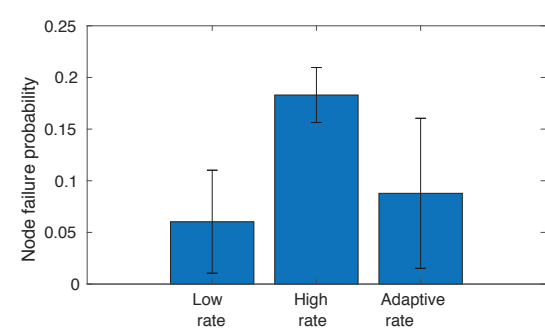

(a) Low-Power Settings

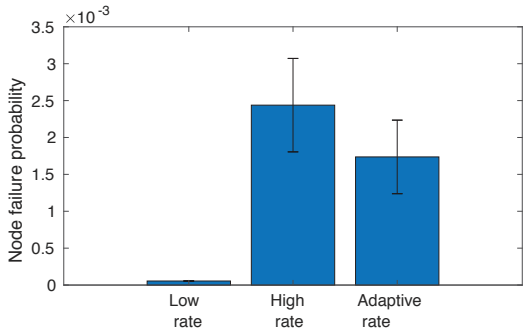

(b) High-Power Settings

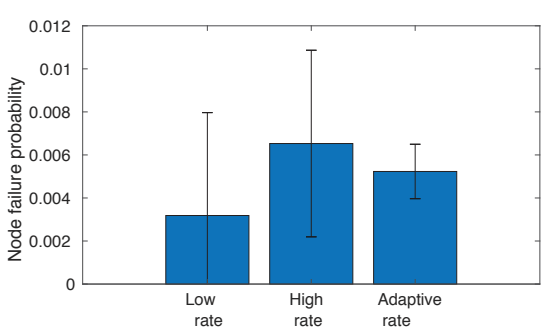

(c) Varying-Power Settings

Figure 16-Availability vs Configuration. The figure plots the median failure probability of a node across different configurations in (a) low-power, (b) high-power, and (c) varying-power settings. The error bars represent the standard deviations.

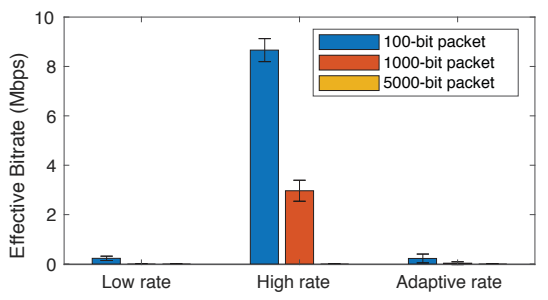

(a) Low-Power Settings

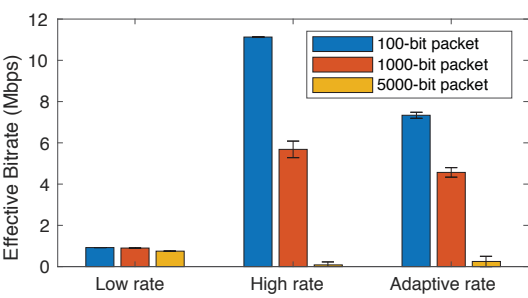

(b) High-Power Settings

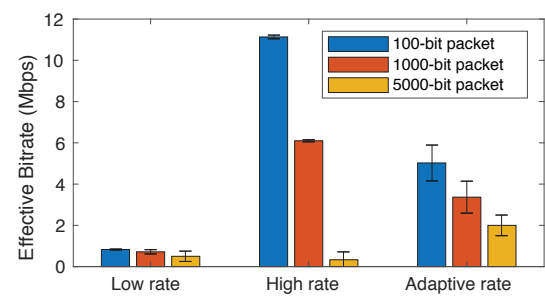

(c) Varying-Power Settings

Figure 17-Throughput vs Configuration. The figure plots the median effective bitrate across configurations in (a) low-power, (b) high-power, and (c) varying-power setting. The error bars represent the standard deviations.

\section{RELATED WORK}

(a) Antenna Reconfigurability. Antenna reconfigurability can refer to a variety of techniques such as beamforming [38], polarization change [58], and frequency tuning [29]. $\mu$ medIC's design is most related to past work on frequency reconfiguration that aims to reuse the same antenna front-end for different frequency bands (e.g., LTE vs 5G). Operating inside the body, however, introduces at least two new unique challenges: first, in contrast to air - which is a homogeneous medium from an RF perspective - the human body is neither homogeneous nor predictable as it consists of different layers of tissues which vary across individuals and across body parts. Second, $\mu$ medIC's design not only needs to shift the antenna itself, but also the entire resonant structure (i.e., antenna+rectifier); which introduces additional complexity to the reconfiguration problem if one wishes to maintain high efficiency as we explained in $\$ 4{ }^{7}$ $\mu$ medIC's architecture allows it to overcome these challenges while maintaining a near-zero power budget and operating inside tissues.

(b) Deep-Tissue RF. $\mu$ medIC's design also builds on recent work on in-body RF backscatter. Recent designs have demonstrated RF backscatter in shallow tissues $[22,63]$ as well as the potential of operating deeper inside the body [33,54]. Such designs, however need to isolate the antenna from nearby tissues by isolating the implant inside a test-tube or by forgoing energy harvesting [33, 54]. Prior work has also explored mechanisms to improve signal-to-noise ratio (SNR) of in-body backscatter [60] but also ignored the impact of surrounding tissues, which resulted in non-FCC compliant behavior. Our work directly advances this line of work and introduces resonance reconfigurability to enable embedding batteryless backscatter micro-implants directly in tissues.

(c) Backscatter Communication. Furthermore, $\mu$ medIC builds on a large and growing literature in backscattering different technologies, such as WiFi, TV signals, and Bluetooth [22, 23, 31, 62]. Our

\footnotetext{
${ }^{7}$ Specifically, in-air antennas can assume $50 \mathrm{Ohm}$ matching, which would not be desirable for high harvesting efficiency [52].
}

contributions are orthogonal and can be combined with these past proposals if one wishes to operate the micro-implants at their other frequency bands (albeit operating at higher frequencies would introduce more attenuation [40]). Prior work has also explored bitrate adaptation for backscatter, primarily in the context of RFIDs [39, 56], but also for ambient backscatter [55]. $\mu$ medIC's rate adaptation is similarly motivated by the desire to reduce overhead, and it enables more efficient rate adaptation by tracking sensor hints in the IC itself.

(d) Non-RF In-Body Wireless. $\mu$ medIC is related to a large body of literature on in-body powering and communication using other approaches such as ultrasound [14], near-field [48], and midfield [21]. These approaches require either direct or near-direct contact with the body, and, as a result, have different application domains than RF-based systems like ours which can operate from larger distances outside the body [33, 63]. Thus, longer-range RF-based systems like $\mu$ medIC would result in more user-friendly implementations and pave way for easier remote and/or mobile healthcare solutions.

\section{CONCLUSION}

This paper presents $\mu$ medIC, a self-reconfiguring fully-integrated wireless platform for batteryless in-body sensors. Our platform employs programmable structures in its antenna, harvesting circuits, and logic. The design is implemented on an IC and tested across tissues to demonstrate its ability to adapt its energy harvesting and backscatter throughput. Looking ahead, this technology paves way for a new generation for networked micro-implants capable of adapting to complex and time-varying in-body conditions.

Acknowledgments. We thank Mohamed Ibrahim for helpful discussions on antenna design and measurements. We also thank the Signal Kinetics group and the anonymous MobiCom reviewers and shepherd for their helpful feedback on the manuscript. This research is supported by an NSF CAREER Award, the MIT Media Lab, and the Endowed Fellowship of the Arab Republic of Egypt. 


\section{REFERENCES}

[1] Agilent N9020A MXA Signal Analyzer. https://www.keysight.com/en/ pdx-x202266-pn-N9020A/mxa-signal-analyzer-10-hz-to-265-ghz?cc=US\&lc= eng.

[2] Ansys HFSS. https://www.ansys.com/products/electronics/ansys-hfss. Ansys High Frequency Structure Simulator (HFSS)

[3] Avery denison. http://rfid.averydennison.com. Avery Denison.

[4] CDA2990. http://www.ettus.com. Ettus Inc.

[5] Flexpcb. https://flexpcb.com/. FlexPCB.

[6] Keysight N5183B MXG Analog Signal Generator. https://www.keysight.com/en/pdx-x202011-pn-N5183B/ mxg-x-series-microwave-analog-signal-generator-9-khz-to-40-ghz?cc= US\&lc=eng.

[7] LFRX daughterboard. http://www.ettus.com. ettus inc.

[8] LP0410. http://www.ettus.com. Ettus Inc.

[9] SBX daughterboard. http://www.ettus.com. ettus inc.

[10] usrp n210. http://www.ettus.com. ettus inc.

[11] Balanis, C. A. Antenna theory: analysis and design; 4th ed. Wiley, Somerset, 2015.

[12] BAO, Z., GuO, Y., AND MitTra, R. Single-layer dual-/tri-band inverted-f antennas for conformal capsule type of applications. IEEE Transactions on Antennas and Propagation 65, 12 (2017), 7257-7265.

[13] Bashirullah, R. Wireless implants. IEEE microwave magazine 11, 7 (2010), S14-S23.

[14] Charthad, J., Weber, M. J., Chang, T. C., And Arbabian, A. A mmsized implantable medical device (imd) with ultrasonic power transfer and a hybrid bi-directional data link. IEEE Journal of solid-state circuits 50, 8 (2015), $1741-1753$.

[15] Chen, X., Zhang, X., Zhang, L., Li, X., Qi, N., Jiang, H., And Wang, Z. A wireless capsule endoscope system with low-power controlling and processing asic. IEEE Transactions on Biomedical Circuits and Systems 3, 1 (2009), 11-22.

[16] Dagdeviren, C. The future of bionic dynamos. Science 354, 6316 (2016), $1109-1109$

[17] Dardari, D., D'Errico, R., Roblin, C., Sibille, A., And Win, M. Z. Ultrawide bandwidth rfid: The next generation? Proceedings of the IEEE 98, 9 (2010), 1570-1582.

[18] Delong, B. J., Kiourti, A., And Volakis, J. L. A radiating near-field patch rectenna for wireless power transfer to medical implants at $2.4 \mathrm{ghz}$. IEEE Journal of Electromagnetics, RF and Microwaves in Medicine and Biology 2, 1 (2018), 64-69.

[19] Gabriel, S., Lau, R. W., And Gabriel, C. The dielectric properties of biological tissues: Iii. parametric models for the dielectric spectrum of tissues. Physics in Medicine \& Biology 41, 11 (1996), 2271.

[20] Gollakota, S., Hassanieh, H., Ransford, B., Katabi, D., and Fu, $\mathrm{K}$. They can hear your heartbeats: non-invasive security for implantable medical devices. In Proceedings of the ACM SIGCOMM 2011 conference (2011), pp. 2-13.

[21] Ho, J. S., Yeh, A. J., Neofytou, E., Kim, S., Tanabe, Y., Patlolla, B., Beygui, R. E., AND PoOn, A. S. Wireless power transfer to deep-tissue microimplants. Proceedings of the National Academy of Sciences 111, 22 (2014), 7974-7979.

[22] Iyer, V., Talla, V., KellogG, B., Gollakota, S., and Smith, J. Intertechnology backscatter: Towards internet connectivity for implanted devices. In Proceedings of the 2016 ACM SIGCOMM Conference (2016), ACM, pp. 356-369.

[23] JANG, J., AND ADIB, F. Underwater backscatter networking. In Proceedings of the ACM Special Interest Group on Data Communication (2019), pp. 187-199.

[24] Jou, A. Y.-S., Pajouhi, H., Azadegan, R., and Mohammadi, S. A cmos integrated rectenna for implantable applications. In 2016 IEEE MTT-S International Microwave Symposium (IMS) (2016), IEEE, pp. 1-3.

[25] KAng, J., ChiAng, P. Y., AND NATARAJAN, A. A $1.2 \mathrm{~cm} 22.4 \mathrm{ghz}$ self-oscillating rectifier-antenna achieving $-34.5 \mathrm{dbm}$ sensitivity for wirelessly powered sensors. In 2016 IEEE International Solid-State Circuits Conference (ISSCC) (Jan 2016), pp. 374-375.

[26] Kang, J., Rao, S., Chiang, P., and Natarajan, A. Design and optimization of area-constrained wirelessly powered cmos uwb soc for localization applications. IEEE Transactions on Microwave Theory and Techniques 64, 4 (April 2016), $1042-1054$.

[27] Keithley Instruments, InC. Model 2400 SourceMeter Instrument, 102018

[28] Kim, J., AND RAHMAT-SAMII, Y. Implanted antennas inside a human body: Simulations, designs, and characterizations. IEEE Transactions on microwave theory and techniques 52, 8 (2004), 1934-1943.

[29] Kolsrud, A. T., Li, M., AND Chang K.. Dual-frequency electronically tunable cpw-fed cps dipole antenna. Electronics Letters 34, 7 (April 1998), 609-611.

[30] Liu, C., Guo, Y.-X., Sun, H., AND XIAO, S. Design and safety considerations of an implantable rectenna for far-field wireless power transfer. IEEE Transactions on antennas and Propagation 62, 11 (2014), 5798-5806.

[31] Liu, V., Parks, A., Talla, V., Gollakota, S., Wetherall, D., and SMITH, J. R. Ambient backscatter: wireless communication out of thin air. ACM
SIGCOMM Computer Communication Review 43, 4 (2013), 39-50.

[32] Lodato, R., Lopresto, V., Pinto, R., AND Marrocco, G. Numerical and experimental characterization of through-the-body uhf-rfid links for passive tags implanted into human limbs. IEEE Transactions on Antennas and Propagation 62, 10 (2014), 5298-5306.

[33] Ma, Y., Luo, Z., Steiger, C., Traverso, G., AND Adib, F. Enabling deeptissue networking for miniature medical devices. In ACM SIGCOMM (2018).

[34] Merli, F., Bolomey, L., Zurcher, J.-F., Corradini, G., Meurville, E., AND SKRIVERVIK, A. K. Design, realization and measurements of a miniature antenna for implantable wireless communication systems. IEEE Transactions on Antennas and propagation 59, 10 (2011), 3544-3555.

[35] Montgomery, K. L., Yeh, A. J., Ho, J. S., Tsao, V., IYer, S. M., Grosenick, L., Ferenczi, E. A., Tanabe, Y., Deisseroth, K., DelP, S. L., ET AL. Wirelessly powered, fully internal optogenetics for brain, spinal and peripheral circuits in mice. nAture methods (2015).

[36] Naderiparizi, S., Hessar, M., Talla, V., Gollakota, S., and Smith, J. R. Towards battery-free $\{\mathrm{HD}\}$ video streaming. In 15th $\{$ USENIX $\}$ Symposium on Networked Systems Design and Implementation (\{NSDI\} 18) (2018), pp. 233247.

[37] Nikolayev, D., Zhadobov, M., And Sauleau, R. Impact of tissue electromagnetic properties on radiation performance of in-body antennas. IEEE Antennas and Wireless Propagation Letters 17, 8 (2018), 1440-1444.

[38] Panagamuwa, C. J., Chauraya, A., And Vardaxoglou, J. C. Frequency and beam reconfigurable antenna using photoconducting switches. IEEE Transactions on Antennas and Propagation 54, 2 (Feb 2006), 449-454.

[39] Pengyu, Z., Jeremy, G., And Deepak, G. Blink: a high throughput link layer for backscatter communication. In Proceedings of the 10th international conference on Mobile systems, applications, and services (New York, NY, USA, 2012), MobiSys '12, ACM, pp. 99-112.

[40] Poon, A. S., O’Driscoll, S., And Meng, T. H. Optimal frequency for wireless power transmission into dispersive tissue. IEEE Transactions on Antennas and Propagation 58, 5 (2010), 1739-1750.

[41] Pozar, D. M. Microwave engineering; 3rd ed. Wiley, Hoboken, NJ, 2005.

[42] RamRakhyani, A. K., Mirabbasi, S., And Chiao, M. Design and optimization of resonance-based efficient wireless power delivery systems for biomedical implants. IEEE Transactions on Biomedical Circuits and Systems 5, 1 (2010), 48-63.

[43] Rosen, A., Stuchly, M. A., And Vander Vorst, A. Applications of rf/microwaves in medicine. IEEE Transactions on Microwave Theory and Techniques 50, 3 (2002), 963-974.

[44] Rostami, M., Gummeson, J., Kiaghadi, A., And Ganesan, D. Polymorphic radios: A new design paradigm for ultra-low power communication. In Proceedings of the 2018 Conference of the ACM Special Interest Group on Data Communication (2018), pp. 446-460.

[45] Sadagopan, K. R., Kang, J., Ramadass, Y., and Natarajan, A. A 960pw co-integrated-antenna wireless energy harvester for wifi backchannel wireless powering. In 2018 IEEE International Solid - State Circuits Conference - (ISSCC) (Feb 2018), pp. 136-138.

[46] SCIENCE, U., AND OFFICE, T. P. Nanotechnology-inspired grand challenges for the next decade. Federal Register (2015).

[47] Seo, D., Neely, R. M., Shen, K., Singhal, U., Alon, E., Rabaey, J. M., CARmena, J. M., AND MAharbiz, M. M. Wireless recording in the peripheral nervous system with ultrasonic neural dust. Neuron 91, 3 (2016), 529-539.

[48] Seo, Y.-S., Hughes, Z., Isom, D., Nguyen, M. Q., Deb, S., Rao, S., AND ChIAO, J.-C. Wireless power transfer for a miniature gastrostimulator. In Microwave Conference (EuMC), 2012 42nd European (2012), IEEE, pp. 229-232.

[49] SKRIVERVIK, A. K. Implantable antennas: The challenge of efficiency. In 2013 7 th European conference on antennas and propagation (EuCAP) (2013), IEEE, pp. 3627-3631.

[50] SON, H. ., AND Pyo, C. . Design of rfid tag antennas using an inductively coupled feed. Electronics Letters 41, 18 (2005), 994-996.

[51] Stoopman, M., Keyrouz, S., Visser, H. J., Philips, K., and Serdijn, W. A. A self-calibrating RF energy harvester generating $1 \mathrm{~V}$ at $-26.3 \mathrm{dBm}$. In 2013 Symposium on VLSI Circuits (2013), pp. C226-C227.

[52] Sun, H., Guo, Y.-X., He, M., AND ZHONG, Z. Design of a high-efficiency 2.45-ghz rectenna for low-input-power energy harvesting. IEEE Antennas and Wireless Propagation Letters 11 (2012), 929-932.

[53] The INDEPENDENT. Swedish workers implanted with microchips to replace cash cards and ID passes, 2017. https://www.independent.co.uk/news/world/europe/sweden-workers-microchipimplant-cash-card-id-pass-replace-employee-hand-epicenter-rice-grain-sizea7670551.html.

[54] Vasisht, D., Zhang, G., Abari, O., Lu, H.-M., Flanz, J., And Katabi, D. In-body backscatter communication and localization. In Proceedings of the 2018 Conference of the ACM Special Interest Group on Data Communication (2018), ACM, pp. 132-146.

[55] Vincent, L., VAmsi, T., AND Shyanmnath, G. Enabling instantaneous feedback with full-duplex backscatter. In Proceedings of the 20th annual international 
conference on Mobile computing and networking (New York, NY, USA, 2014), MobiCOM '14, ACM, pp. 67-78.

[56] Wei, G., Si, C., JiAngChuAn, L., AND ZHI, W. Mobirate: Mobility-aware rate adaptation using phy information for backscatter networks. In IEEE Conference on Computer Communications (New York, NY, USA, 2018), INFOCOM'18, IEEE, pp. $1259-1267$

[57] WEI, T., AND ZHANG, X. Gyro in the air: tracking $3 \mathrm{~d}$ orientation of batteryless internet-of-things. In Proceedings of the 22nd Annual International Conference on Mobile Computing and Networking (2016), pp. 55-68.

[58] Wong, H., Lin, W., Huitema, L., And ARnaud, E. Multi-polarization reconfigurable antenna for wireless biomedical system. IEEE transactions on biomedical circuits and systems 11, 3 (2017), 652-660.

[59] Xue, R.-F., CHEng, K.-W., AND JE, M. High-efficiency wireless power transfer for biomedical implants by optimal resonant load transformation. IEEE Transactions on Circuits and Systems I: Regular Papers 60, 4 (2012), 867-874.
[60] Yakovlev, A., Jang, J. H., And PivonkA, D. An $11 \mu \mathrm{wsub}-\mathrm{pj} / \mathrm{bit}$ reconfigurable transceiver for $\mathrm{mm}$-sized wireless implants. IEEE Transactions on Biomedical Circuits and Systems 10, 1 (Feb 2016), 175-185.

[61] Yuce, M. R., AND DissanaYAKe, T. Easy-to-swallow wireless telemetry. IEEE Microwave magazine 13, 6 (2012), 90-101.

[62] Zhang, P., Bharadia, D., Joshi, K., and Katti, S. Hitchhike: Practical backscatter using commodity wifi. In Proceedings of the 14th ACM Conference on Embedded Network Sensor Systems CD-ROM (2016), ACM, pp. 259-271.

[63] Zhang, P., Rostami, M., Hu, P., And Ganesan, D. Enabling practical backscatter communication for on-body sensors. In Proceedings of the 2016 ACM SIGCOMM Conference (2016), ACM, pp. 370-383. 